\title{
BELT CASTING TECHNOLOGY - EXPERIENCES BASED ON THE WORLDWIDE FIRST BCT CASTER*
}

\author{
Jochen Wans ${ }^{1}$ \\ Christian Geerkens ${ }^{2}$ \\ Hermann Cremers ${ }^{3}$ \\ Ulrich Grethe ${ }^{4}$ \\ Peter Juchmann ${ }^{5}$ \\ Rune Schmidt-Jurgensen ${ }^{6}$
}

\begin{abstract}
BCT® - Belt Casting Technology is the new horizontal near-net-shape casting process to produce flat steel products. BCT is the revolutionary leap in casting technology, designed for the production of special and conventional steel grades. Within a partnership between Salzgitter Flachstahl GmbH and SMS Siemag AG, the worldwide first caster is now in operation. SMS Siemag AG (in cooperation with Salzgitter Flachstahl $\mathrm{GmbH}$ ) has erected the first industrial-scale casting machine located at Peine steelworks. The lecture will start with an overview about the highlights of the $\mathrm{BCT} \circledast$ process with regard to the mechanical engineering aspects, while the second part will present current commissioning results and operation experiences. Since December 2012, the BCT plant is running. Up to March 2015 more than 90 casts have been carried out, 26 with FeMn-alloys. Never before flat steel was casted in dimensions of $1000 \mathrm{~mm}$ * $15 \mathrm{~mm}$ and with casting speeds more than $14 \mathrm{~m} / \mathrm{min}$.
\end{abstract}

Keywords: Belt casting technology, BCT®; Strip casting; High-alloy steel grades; Steel properties.

1 Dr.-Ing. Metallurgy, General Manager, Technical Sales Special Technologies / Development, SMS Siemag AG, Eduard-Schloemann-Str. 4, D-40237 Düsseldorf, Germany.

2 Dipl.-Ing. Electrotechnology, Executive Vice President, Metallurgical Plants and Environmental Division, SMS Siemag AG, Germany.

3 Dipl.-Ing. Metallurgy, Commissioning Engineer, Commissioning/Process Technology, SMS Siemag AG, Germany.

4 Dipl.-Ing. Metallurgy, Chairman of the Board of Directors, Salzgitter Flachstahl GmbH, Germany.

5 Dr.-Ing. Mechanical Engineering, Head of belt casting / hot rolling, Salzgitter Flachstahl GmbH, Germany.

6 Dr.-Ing. Mechanical Engineering, Plant manager belt casting, Salzgitter Flachstahl GmbH, Germany. 


\section{INTRODUCTION}

Unlike many other materials, steel is subject to ever increasing requirements. Hightech components made of modern steels already offer a variety of applications. In the future the manufacture of innovative products requires the availability of steel grades with significantly improved properties.

Steelmakers are therefore faced with the challenge of developing new steel alloys that satisfy the specific requirements. However, the production, use and recycling of steel today must also comply with environmental standards.

Especially high and ultra-high alloy steel concepts for use in the manufacture of lightweight automobiles, offshore applications or in the chemical industry are needed. Where these alloys are used, they combine optimal mechanical properties and thus present steelmakers and their existing plant and equipment with new challenges [1].

Plant and equipment builders must develop suitable plant and processes for their customers. The focus here is on the further development of existing processes which offer customers a financial benefit. One example of this is SMS Siemag's CSP® technology which has been existing for more than 20 years. Another main area is the development of novel plant concepts.

The newly developed plant concept based on Belt Casting Technology (BCT®) is now realized by SMS Siemag for the first time on an industrial scale.

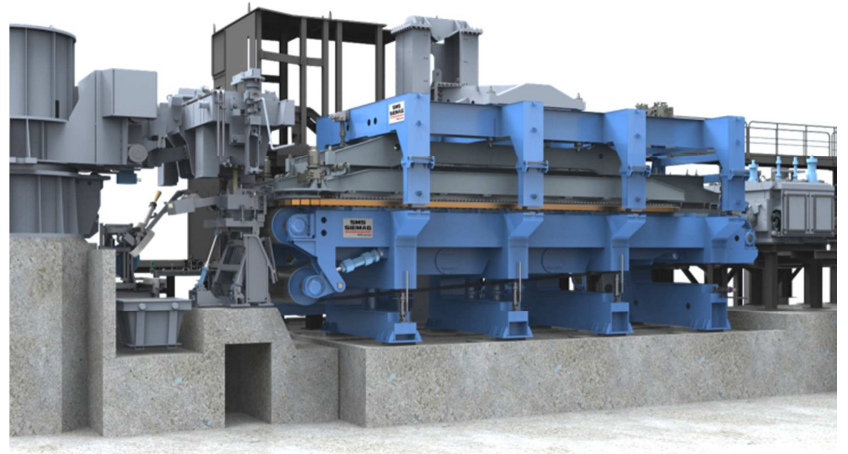

Figure 1. BCT® caster

In a cooperation project with Salzgitter Flachstahl $\mathrm{GmbH}$ this plant is used to implement the near-net-shape casting of $\mathrm{HSD}{ }^{\circledR}$ steel grades. Main technological expertise has been developed together with Clausthal University. The mechanical engineering of SMS Siemag presents the basis for the commercial launch of this forward-looking casting technology. SMS Siemag thus will extend its portfolio of custom-made and energy-efficient casting and rolling installations.

The revolutionary Belt Casting Technology is another technology leap on the way to near-net-shape casting. BCT® casters will allow different production rates and a large variety of product sizes, depending on the desired plant concept. The product line for BCT® casters will mainly comprise alloys that so far could not be continuously cast, as well as crack-critical conventional steel grades.

With the present paper the cooperation partner wish to highlight the advantages of the technology and the commissioning progress of the first industrial-scale BCT® caster.

(HSD® is a registered brand of Salzgitter Flachstahl GmbH.). 


\section{MATERIAL AND METHODS}

\subsection{BCT $\circledast$ - The Technology Leap}

The near-net-shape horizontal belt casting of steel opens up new technological possibilities.

Many of the steel grades which presently entail yield losses in conventional plants are expected to be profitably produced in BCT® installations. Steels with extraordinary properties can now for the first time be produced on an industrial scale: $\mathrm{HSDB}$ steel [2] with its high contents of manganese, silicon and aluminum: lightweight, high strength and at the same time easily deformable.

All is made possible by casting metal onto the moving mold without the addition of casting flux, as well as stress-free horizontal solidification of the as-cast strip.

The rapid solidification in an inert atmosphere opens up a wide range of potential charge materials [3]. The caster may be combined both with BOF and electric arc furnace melt shops. BCT® installations may be specifically tailored to the needs of SMS Siemag's customers. Medium-wide strip can be produced just as well as hot wide strip.

Customers with small production capacities will be served by the appropriate installation, as well steel producers with medium-to-high annual tonnages.

The caster will be designed for the necessary metallurgical length and casting speed. Compact BCT® casting-rolling installations produce $15 \mathrm{~mm}$ as-cast strip - a near-netshape thickness that allows both a sufficient deformation degree to attain optimal mechanical properties and saves deformation energy, space and eventually cost..

\subsection{The First Industrial-scale BCT® Caster}

In May 2010, Salzgitter Flachstahl awarded SMS Siemag a contract for the construction of the world's first horizontal, industrial-scale BCT® caster.

Utilizing existing infrastructure, a financial first-plant concept could be drawn up in cooperation with Salzgitter, whose focus is on testing of the casting technology. SMS Siemag's wealth of experience in the planning, construction and trial operation of the pilot plants set up at the MEFOS Research Institute in Sweden as well as at the technological competence of Technical University of Clausthal are essential factors that contributed to the development and design of the new machine. The laboratory strip caster at the Institute of Metallurgy of the Technical University of Clausthal was used to successfully cast the novel HSD® steel grades.

The new BCT® caster is installed at Peiner Träger $\mathrm{GmbH}$ (Salzgitter Group) steelworks (Fig. 2). The HSD® steel grades on which the focus is here are made using the SMS Siemag supplied and commissioned units comprising electric arc furnace with ARCCESSß-technology, twin-type ladle furnace and vacuum degasser. The BCT® caster produces $15 \mathrm{~mm}$ thick and $1,000 \mathrm{~mm}$ wide material. Essential parts of the plant are already rated for a width of $1,600 \mathrm{~mm}$ to allow the future extension of the product line.

At the Salzgitter works, an existing four-high mill stand was upgraded to allow the further processing of the as-cast strip produced in Peine.

SMS Siemag supplied the Basic and Detail Engineering, all mechanical plant components including the complete X-Pact® electrical and automation package and erected the equipment for the $\mathrm{BCT}{ }^{\circledR}$ caster and the modernization of the mill stand. 


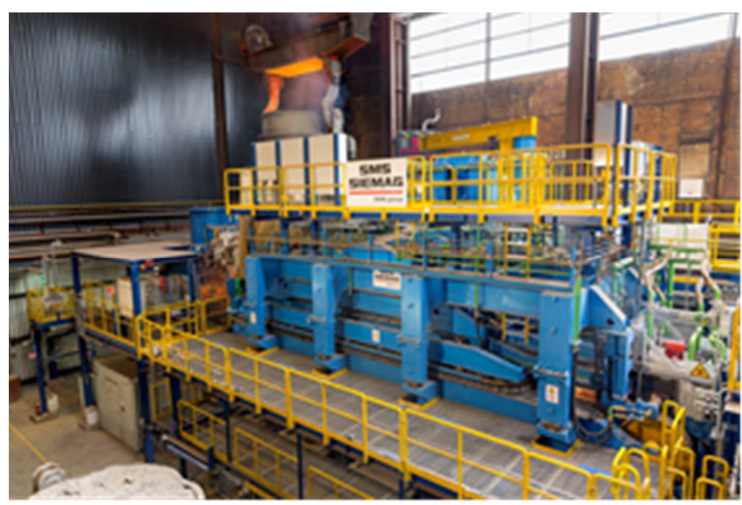

Figure 2. Set-up of $B C T \AA$ caster in Peine.

In December 2012, the cooperation partners Salzgitter Flachstahl and SMS Siemag did jointly commission the facilities in order to implement the benefits of this technology on an industrial scale.

Footprint of the machine is approx. $11 \mathrm{~m} * 4 \mathrm{~m}, 6 \mathrm{~m}$ tall. Machine length is owing to the solidification range of special steel grades.

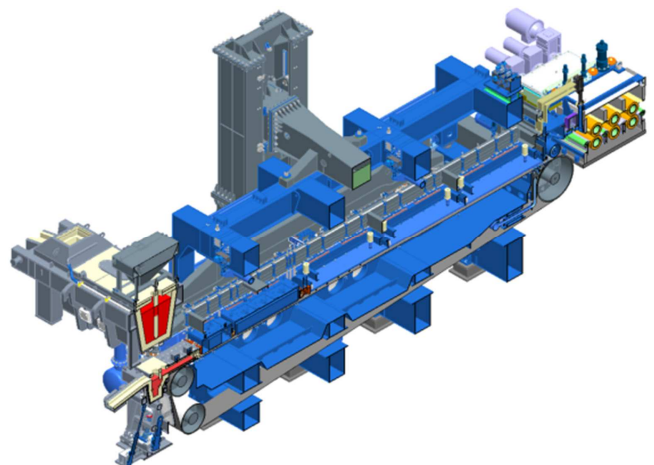

Figure 3. BCT® caster, longitudinal section.

Protected from reoxidation, the liquid steel is poured from a conventional ladle into a covered tundish and from there, via the submerged entry nozzle, into the preheated feeding system. From this system the steel flows onto the moving horizontal mold which consists of the conveyor belt and the internally cooled side dams.

The underside of the conveyor belt is intensively cooled by water. No oscillating mold is therefore needed for initial solidification, no addition of casting flux is required.

Above the conveyor belt an inert gas atmosphere protects the solidifying steel. At the rear end of the caster, a defined gas mixture directly influences the solidification structure.

Electro-magnetic units to influence the steel flow as well as cooled hoods are arranged above the conveyor belt. A transverse stirrer supports the smooth and uniform distribution of the liquid steel up to the side dams. A longitudinal stirrer synchronizes the movement of the conveyor belt with the liquid steel flow. This stirring technology is the result of a joint development. SMS Elotherm GmbH delivered both units.

The as-cast strip leaves the conveyor belt horizontally. It is guided by a top roller and three pairs of ironing rollers that can influence the flatness of the strip. Downstream these rollers both the strip thickness and profile are measured. The as-cast strip then enters an enclosed roller table in which the complete inertization of the process is ensured up to the end of the table. 
The roller table is followed by a combination of two pinch-roll stands and a looper arranged in between. In addition to the further transport of the as-cast strip, it makes sure that influences which downstream units have on the as-cast strip are decoupled. The second pinch roll unit feeds the strip to a moving hydraulic shear. For cutting, the shear is accelerated to the as-cast strip speed, cutting it to the desired sheet length.

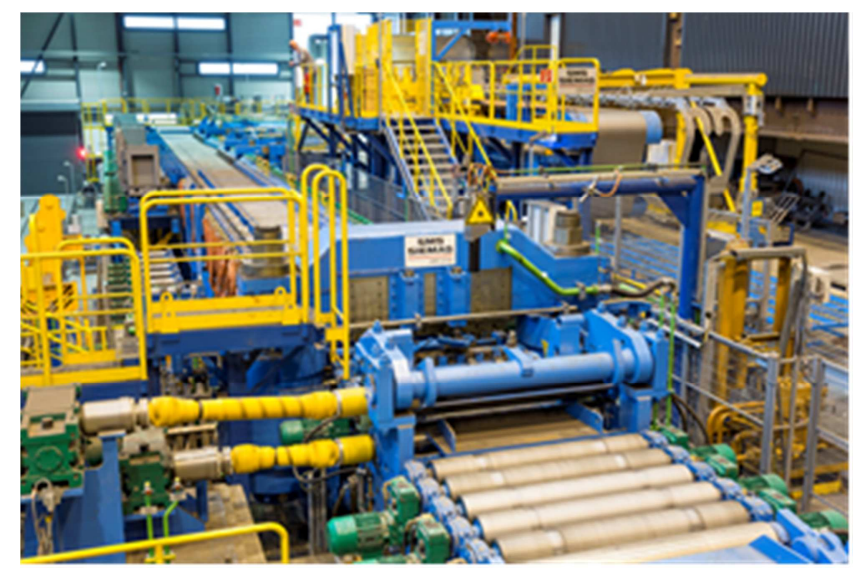

Figure 4. Shear and roller table.

A roller table accelerates the sheets which here have a maximum length of $9 \mathrm{~m}$; towards the end of the roller table the sheets are decelerated again. Cross-pushers place the sheets on a hydraulically operated lifting table. The sheets stacked on pallets are loaded onto wagons and taken to the rolling mill in Salzgitter..

\section{RESULTS AND DISCUSSION}

\subsection{BTC Plant Service Experiences Obtained from Hot Commissioning up to Now}

In December 2012, Salzgitter Flachstahl and SMS Siemag started the hot commissioning of the belt casting plant. The maximum steel quantity of approx. 70 tons, for which this first BCT plant has been de-signed, was already casted a few weeks later in a reproducible and safe manner. Due to the close co-operation of both partners, a continuous improvement in equipment and process performance could be achieved. The principle robustness and reliability of alloy production, melt transfer and belt casting have been confirmed by using conventional carbon steel grades. The performance of important machine components, such as the casting nozzle, the conveyor belt and the moving side dams has been satisfactory. At the start of commissioning, only carbon steel was casted but meanwhile the casting program has been enhanced by high-manganese steel grades. Up to March 2015 more than 90 casts would have been carried out, including 26 times FeMn steel grades. Figure 5 shows the belt casting plant in production process. The as-cast strip exits the cooling zone behind the caster. 


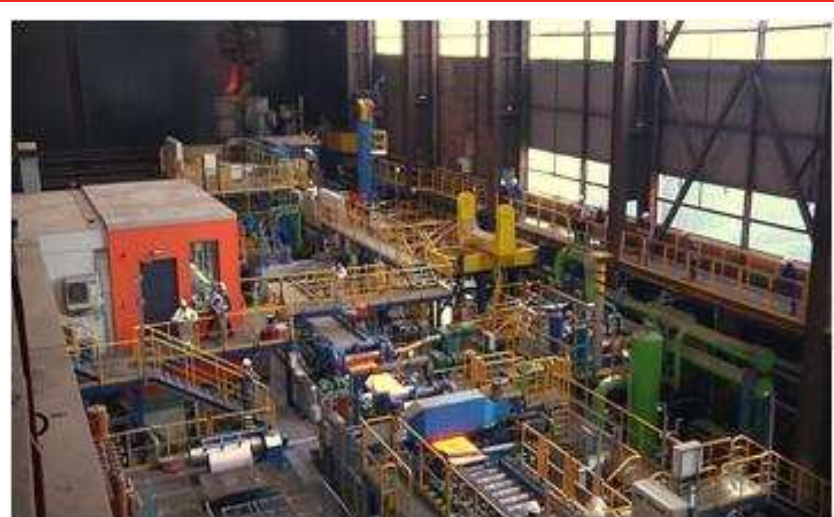

Figure 5. Belt casting plant in production process.

Meanwhile belt cast carbon steel has been success-fully hot rolled down to a thickness of 2.5 to $5 \mathrm{~mm}$, coiled and tested in downstream process steps [4]. The technical properties allow for first commercial applications (Figure 6).

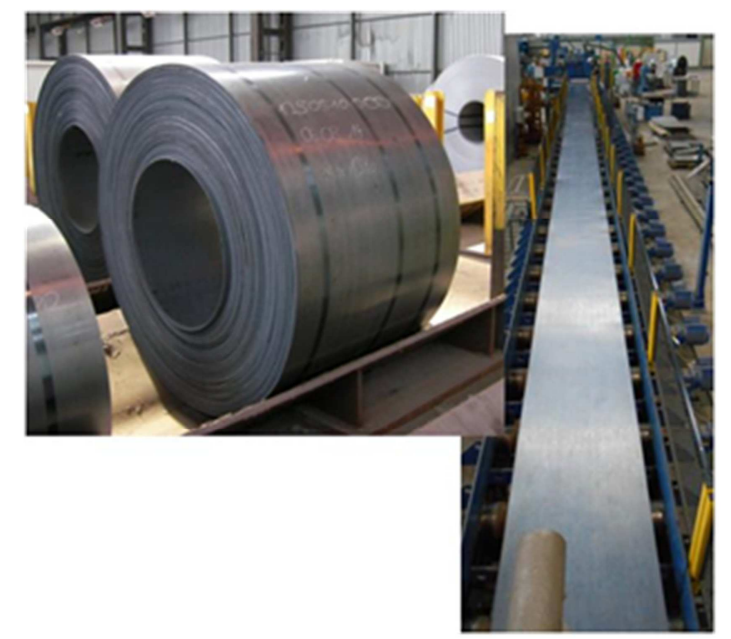

Figure 6. Hot rolled carbon steel strip $(2.5 \mathrm{~mm})$ after laminar cooling on the exit roller table of the rolling mill (right); Hot strip coils build up by plasma welding of several strip pieces (left).

Beside carbon steel, complete FeMn melts could also be casted safely several times. Then the regular casting time amounts to roughly 40 minutes. Figure 7 shows the as cast strip of a FeMn steel grade. A total product length of about $600 \mathrm{~m}$ is passing the shear to be cut into $9 \mathrm{~m}$-long plates.

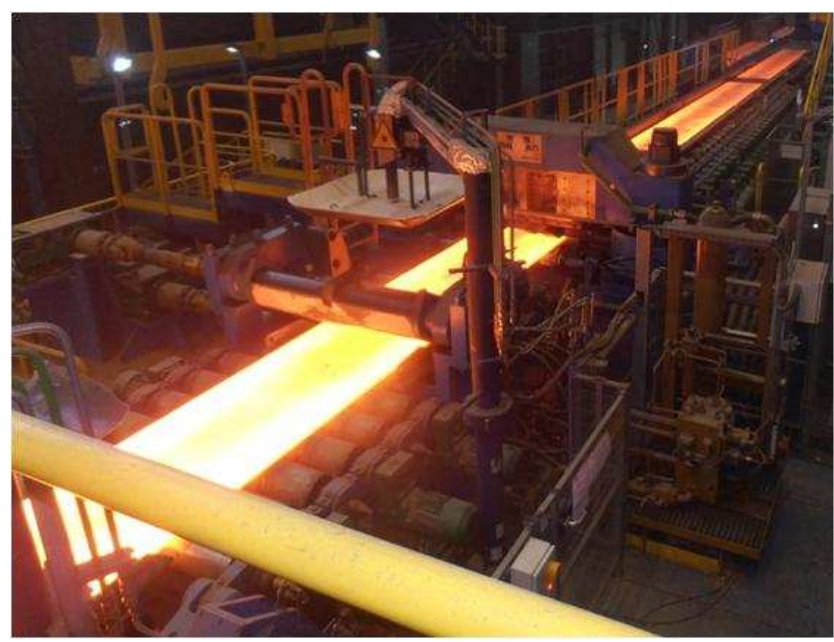

Figure 7. As-cast strip (FeMn-grade) passing the shear. 
The current process development and plant operation are focused on further adapting the belt casting process to FeMn steel grades in order to improve the ascast strip quality for hot rolling. Main challenges refer to an equilized melt distribution and as-cast profile respectively. The product quality also depends on a powerful inertization from the tundish to the downstream cooling zone behind the caster and optimized cooling conditions.

\section{CONCLUSION}

The newly developed plant concept based on Belt Casting Technology (BCT®) is now realized by the plant builder SMS Siemag AG for the first time on an industrial scale. In a cooperation project with Salzgitter Flachstahl $\mathrm{GmbH}$ this plant is used to implement the near-net-shape casting and rolling of $H S D \AA$ steel grades.

During the first experiences of the commissioning the BCT® caster produces $15 \mathrm{~mm}$ thick and $1,000 \mathrm{~mm}$ wide as-cast strip. The product mix leads from medium carbon grades up to high Manganese steel grades with Aluminum and Silicon. The results achieved up to today are highly promising.

The Belt Casting Technology enables the production of new steel alloys, with horizontal strip casting offering an opportunity to produce steel grades which in conventional installations can be cast to a limited extent only. BCT $\AA^{\circ}$ is characterized by stress-free solidification, with no casting flux required for this process. Together with Clausthal University the basis of the process technology has been developed.

$B C T \AA$ installations are flexible when it comes to their integration in new or existing BOF or electric arc furnace melt shops. The production capacity and product dimensions are designed specifically for each customer.

As future outlook we are concretizing a first endless concept (casting and rolling in one line) according to the request of one of our customers.

\section{REFERENCES}

1 Gigacher, G., Bernhard, C., Kriegner, W.: Eigenschaften hochmanganhaltiger Stähle unter stranggießähnlichen Bedingungen, BHM, 149 (2004) No. 3, pp. 112-117.

2 Kämper, S.: Forschungsinnovation für den Kunden, Stahl und Eisen 129 (2009) Nr. 6

3 Spitzer, K.-H., Rüppel, F., Viscorova, R., Scholz, R., Kroos, J., Flaxa, V.: Direct Strip Casting - an Option for the Production on New Steel Grades, steel research 74 (2003) No. 11/12, pp. 724-731

4 Wans, J; Geerkens, Ch.; Cremers, H.; Grethe, U.; Juchmann, P.; Schmidt-Jürgensen, R.; Belt Casting Technology $(\mathrm{BCT} \AA)$ - Process technology and operating results of the worldwide first industrial BCT®-caster, Stahl 2014 